\title{
Value of Neutrophil Lymphocytic Ratio and Platelet Lymphocytic Ratio in Premature Rupture of Membranes for Detection of Subclinical Chorioamnoitis
}

\author{
Shereen B Elbohoty ${ }^{1 *}$, Shereef elshwaikh ${ }^{1}$,Heba Harras ${ }^{2}$ and Amal Elsokary ${ }^{1}$ \\ ${ }^{1}$ Lecturer of Obstetrics and Gynecology, Faculty of Medicine, Tanta University, Egypt \\ ${ }^{2}$ Lecturer of pathology, Faculty of Medicine, Tanta University, Egypt
}

*Corresponding author: Shereen B Elbohoty, Lecture of obstetrics and gynecology,

Tanta University, Tanta, 31111, Egypt.

Received Date: February 03, 2020

Published Date: February 13, 2020

Abstract

Aim: To evaluate neutrophil lymphocytic ratio (N/L) and platelet lymphocytic (P/L) ratio in detection of subclinical Chorioamnoitis in pregnant females suffering from premature rupture of membranes and to compare them with CRP and TLC.

Design: Clinical randomized controlled study.

Methods: 100 pregnant patients were selected suffering from PROM , and prepared for termination of pregnancy either normal or cesarean , all had no clinical signs of chorioamnoitis , blood markers ( CRP, TL, N/L ratio and P/L ratio) were taken before termination, the results statistically analyzed according to the result of pathological examination of amniotic membrane for detection of early inflammatory signs for chorioamnoitis.

Results: There was significant relationship for the P/L ratio and the finding of early chorioamnoitis by pathological examination with $\mathrm{P}$ value 0.0004 , however other markers failed to show any significant relationship with early chorioamnoitis , But CRP was found to have a significant relationship with the presence of postpartum complications with $\mathrm{P}$ value 0.0003 and other markers had insignificant relationship, There was insignificant relationship with all the four markers with the method of delivery , CRP and TLC had lower specificity in detection of subclinical chorioamnoitis as compared by N/L ratio and P/L ratio, also P/L ratio had the higher sensitivity $85.71 \%$, so by comparing the four markers $\mathrm{P} / \mathrm{L}$ ratio is the most accurate $90 \%$ and N/L ratio had accuracy more than TLC and CRP ( $80 \%$ ).

Conclusion: $\mathrm{P} / \mathrm{L}$ ratio and N/L ratio are available cheap markers for detection of subclinical chorioamnoitis, and they show more specificity and accuracy than CRP and TLC in detection of subclinical chorioamnoitis, also P/L ration had higher sensitivity.

Keywords: Subclinical chorioamnoitis, PROM , N/L ratio , P/L ratio , CRP , TLC

\section{Introduction}

Amniotic membrane, which is considered as a closed envelope around the fetus, is the most important barrier for protecting fetus from exterior, and the contained amniotic fluid gives the fetus the space to move and a media for excretion and also nutrion [1], Amniotic sac should remain intact till near the end of second stage of labour , Any break in the sac before that should be considered as a premature rupture of membrane (PROM), if it occurred before 36 weeks gestation it will be considered as preterm premature rupture of membranes [2].
The second most common cause of preterm labour is preterm PROM, as rupture of membranes will lead to local release of inflammatory mediators which in turn lead to premature uterine contractions that may end to preterm labour with its all hazards to the fetus [3].

But the most dangerous and most important concern about preterm PROM is the occurrence of chorioamnoitis [4]. Chorioamnoitis is inflammation of fetal membranes and may 
proceed to underlying decidua, it may lead to maternal toxemia and even septic shock, with very bad fetal outcome [5].

Occurrence of chorioamnoitis should be excluded in every case suffering from PROM, as it has dangerous consequences, and also may affect the course of labour when decision of labour is taken, as the uterus may not respond efficiently to uterotonic drugs in case of vaginal delivery that lead to increase rate of cesarean section, which also may complicated with surgical infection with all its hazards , with increase susceptibility to atonic postpartum hemorrhage [6].

Diagnosis of subclinical infection in case of PROM is a medical challenge. The most important and widely used markers are CRP and TLC , both had accepted specificity but low sensitivity making its use alone had many disadvantages and many misdiagnosed cases [7].

Neutrophil lymphocytic ratio (N/L) and platelet lymphocytic ratio $(\mathrm{P} / \mathrm{L})$ had been suggested to be used as alternative markers [8].

\section{Methods}

Study design: Prospective cohort study

Setting: Tanta University Hospital

Number of cases: 100 patients.

Timing of the study: from jun. 1, 2018 to august,31, 2019.

Cases selection: The cases were selected from the pregnant females whom attending Tanta University Hospital,

They were selected according to the following criteria:

1. Pregnant females with gestational age above 20 weeks gestation

2. Suffering from premature rupture of membranes

3. All the patients selected at time of termination of pregnancy whatever indication is except for acute chorioamnoitis ( like lung maturity, preterm labor pain, PROM before 24 weeks gestation)

And they were excluded if:

1. Presence of any clinical sign of chorioamnoitis ( fever , offensive vaginal discharge, tender uterus and non-reassuring non stress test)

2. If vaginal termination of pregnancy took more than 24 hours.

3. Patient with multiple gestations

4. Any systemic disease may affect $\mathrm{N} / \mathrm{L}$ ratio or $\mathrm{P} / \mathrm{L}$ ratio like Hematological disorders ,Malignancy, Hepatic diseases, autoimmune disease and Chronic renal diseases

5. Gestational D.M and pre-eclampsia.

6. Acute or chronic infectious or inflammatory diseases.

\section{Cigarette smoker}

Sample size calculation: The sample size was calculated using Epi-Info 7 specific program.

\section{Methods:}

- Written consent was taken from all patients submitted to the study with clarification of the methods, value and hazards of the study.

- Detailed history taking from all patients

- All patients before pregnancy termination were evaluated for absence of signs of acute chorioamnoitis

- Then blood sample was taken from the patient at time of termination of pregnancy if termination was normal, and before induction of anesthesia if termination was cesarean section, the blood sample used for :

1. C-reactive protein done by latex agglutination test. We took $2 \mathrm{mg} / \mathrm{dl}$ as a cut off value

2. Another $2 \mathrm{ml}$ of venous blood was collected into an EDTA contained bottle for .CBC measuring using an automated blood counter(ERMA PCE-210N) to measure the following :

A. Total leucocytic count ( normal 4000-11000/ul)

B. Platelet count (PCT), lymphocyte count and neutrophilic count were recorded so P/L ratio will be calculated; we took 125 as a cut off value.

C. Neutrophil -lymphocyte ratio(NLR) was calculated as absolute neutrophil count is divided by absolute lymphocyte count. We took 2.5 as a cut off value.

- Then after successful delivery of the baby, multiple samples from amniotic membrane were taken and sent for pathological examination for confirmation or exclusion of presence of inflammatory reaction so diagnosis of subclinical chorioamnoitis may or may not excluded.

- Correlation of the blood test with the pathological findings of absence or prescience of subclinical chorioamnoitis was evaluated using suitable statistical methods.

- Also, evaluation of the uterus and female postpartum was assessed for any complication.

\section{Outcome:}

\section{A. Primary:}

- Value of $\mathrm{N} / \mathrm{L}$ ration and $\mathrm{P} / \mathrm{L}$ ratio for diagnosis of subclinical chorioamnoitis and compared with CRP and TLC

\section{B. Secondary:}

- Relation of $\mathrm{N} / \mathrm{L}$ ration and $\mathrm{P} / \mathrm{L}$ ratio with timing of termination of pregnancy and compared with CRP and TLC

- $\quad$ Relation of N/L ration and $\mathrm{P} / \mathrm{L}$ ratio with method of termination of pregnancy and compared with CRP and TLC 
- $\quad$ Relation of N/L ration and P/L ratio with postpartum condition of mother and the uterus and compared with CRP and TLC

Ethical approval: This study was approved by local ethical committee of Tanta University before the start of this study.

\section{Results}

100 patients were selected in the course of study, all were suffering from PROM, and prepared for termination of pregnancy either normal or cesarean, all had no clinical signs of chorioamnoitis, blood markers were taken before termination , and the results statistically analyzed according to the result of pathological examination of amniotic membrane for detection of early inflammatory signs for chorioamnoitis.

Studying of patients' data were demonstrated in Table 1, the gestational age at which termination of pregnancy was decided in the cases recruited for the study were range from 22-39 weeks gestation with mean 33.8 weeks, and the period of rupture of membranes range from 1-14 days with mean 5.05 days, of course in some cases the period of rupture of membranes was less than 1 day, those patient the duration of rupture of membrane was approximated to be 1 day.

Table1: Shows distribution of cases according demographic data, period of membranes rupture and blood makers.

\begin{tabular}{|c|c|c|}
\hline \multirow{2}{*}{ Age } & No :100 & \\
\cline { 2 - 3 } & Range & Mean \pm S. D \\
\hline \multirow{2}{*}{ Gravidity } & $20-41$ & 28.25 \\
\cline { 3 - 3 } & & 5.711 \\
\cline { 3 - 3 } & \multirow{2}{*}{$1-5$} & 2 \\
\hline
\end{tabular}

\begin{tabular}{|c|c|c|}
\hline \multirow[t]{2}{*}{ Parity } & \multirow[t]{2}{*}{$0-4$} & 0.947 \\
\hline & & 1.078 \\
\hline \multirow[t]{2}{*}{ BMI } & \multirow[t]{2}{*}{$21-32$} & 26.2 \\
\hline & & 3.071 \\
\hline \multirow[t]{2}{*}{ Gestational age } & \multirow[t]{2}{*}{$22-39$} & 33.8 \\
\hline & & 5.105 \\
\hline \multirow[t]{2}{*}{$\begin{array}{l}\text { Period of rupture of } \\
\text { membranes }\end{array}$} & \multirow[t]{2}{*}{ 1-14 days } & 5.05 \\
\hline & & 3.649 \\
\hline \multirow[t]{2}{*}{ CRP } & \multirow[t]{2}{*}{$1-8$} & 1.706 \\
\hline & & 2.912 \\
\hline \multirow[t]{2}{*}{ TLC } & \multirow[t]{2}{*}{$4-15$} & 9.328 \\
\hline & & 3.631 \\
\hline \multirow[t]{2}{*}{$\mathrm{N} / \mathrm{L}$ ratio } & \multirow[t]{2}{*}{$1.8-2.9$} & 2.345 \\
\hline & & 0.305 \\
\hline \multirow[t]{2}{*}{$\mathrm{P} / \mathrm{L}$ ratio } & \multirow[t]{2}{*}{$115-138$} & 123.2 \\
\hline & & 9.305 \\
\hline
\end{tabular}

There was significant relationship for the P/L ratio and the finding of early chorioamnoitis by pathological examination (which was found in positive in $35 \%$ of cases) with P value 0.0004 , suggesting that $\mathrm{P} / \mathrm{L}$ ratio may have a significant role in detection of subclinical chorioamnoitis, however other markers like CRP , TLC and N/L ratio failed to show any significant relationship with early chorioamnoitis detected by pathological examination (Table 2).

But CRP was found to have a significant relationship with the presence of postpartum complications with P value 0.0003 (15\% of cases had postpartum infection which was in all cases were just surgical wound infection, and $5 \%$ of cases had postpartum atony and all cases were managed successfully by conservative medical treatment) and other markers had insignificant relationship (Table 2).

Table 2: Shows the method of termination, subclinical chorioamnoitis by pathological examination and postpartum results and its relations to blood markers.

\begin{tabular}{|c|c|c|c|c|c|c|c|c|c|c|}
\hline & & & & & & & & & & \\
\hline & $\begin{array}{l}\text { Chi-squal } \\
\text { P value } \\
\text { Chi-squa }\end{array}$ & & $P$ value & $\begin{array}{l}\text { Chi- } \\
\text { square }\end{array}$ & $P$ value & $\begin{array}{l}\text { Chi- } \\
\text { square }\end{array}$ & $P$ value & & & \\
\hline $\begin{array}{l}\text { Method of } \\
\text { termination }\end{array}$ & CS & NVD & & & & & & & & \\
\hline & No: 55 & No :45 & 2.155 & 0.142 & 1.174 & 0.279 & 1.684 & 0.194 & 0.02 & 0.888 \\
\hline & $55 \%$ & $45 \%$ & & & & & & & & \\
\hline $\begin{array}{l}\text { Pathological } \\
\text { chorioam- }\end{array}$ & Yes & No & 0.036 & 0.848 & 0.292 & & 5.934 & 0.0148 & 12.17 & \\
\hline & No :35 & No :65 & & & & 0.588 & & & & $0.0004 *$ \\
\hline & $35 \%$ & $65 \%$ & & & & & & & & \\
\hline
\end{tabular}




\begin{tabular}{|c|c|c|c|c|c|c|c|c|c|c|c|}
\hline $\begin{array}{l}\text { Postpartum } \\
\text { complica- } \\
\text { tions }\end{array}$ & No & Atony & infection & 12.76 & $0.0003^{*}$ & 0.495 & 0.481 & 0.547 & 0.244 & 3.517 & 0.0607 \\
\hline & No: 80 & No: 5 & No: 15 & & & & & & & & \\
\hline & $80 \%$ & $20 \%$ & & & & & & & & & \\
\hline
\end{tabular}

There was insignificant relationship with all the four markers with the method of delivery (55\% of cases delivered by CS and mostly the indication of cesarean section was previous cesarean scar) ( Table 2).

CRP and TLC had lower specificity in detection of subclinical chorioamnoitis proved by histopathological examination of amniotic membranes as compared by N/L ratio and $\mathrm{P} / \mathrm{L}$ ratio, also $\mathrm{P} / \mathrm{L}$ ratio had the higher sensitivity $85.71 \%$, so by comparing the four markers $\mathrm{P} / \mathrm{L}$ ratio is the most accurate $90 \%$ and $\mathrm{N} / \mathrm{L}$ ratio had accuracy more than TLC and CRP ( 80\%) (Table 3).

Table 3: Shows the sensitivity, specificity, positive predictive value, negative predictive value and accuracy of TLC , CRP , N/L ratio and P/L ratio in detection of subclinical chorioamnoitis.

\begin{tabular}{|c|c|c|c|c|c|}
\hline Accuracy & NPV & PPV & Specificity & \multicolumn{2}{|c|}{ Sensitivity } \\
\hline $55 \%$ & $66.67 \%$ & $37.50 \%$ & $61.54 \%$ & $42.86 \%$ & CRP \\
\hline $60 \%$ & $69.23 \%$ & $42.86 \%$ & $69.23 \%$ & $42.86 \%$ & TLC \\
\hline $80 \%$ & $80 \%$ & $80 \%$ & $92.13 \%$ & $57.14 \%$ & N/L ratio \\
\hline $90 \%$ & $92.31 \%$ & $82.71 \%$ & $88.68 \%$ & $85.71 \%$ & P/L ratio \\
\hline
\end{tabular}

\section{Discussion}

Premature rupture of membranes (PROM), affects approximately $3 \%$ of all pregnancies. It is closely related with significant maternal and fetal morbidity and mortality. PROM is one of the most common causes of preterm delivery and is associated with maternal and neonatal infections [2].

The most important complication of PROM is chorioamnoitis which should be avoided by prophylactic antibiotic and timely termination of pregnancy, diagnosis of subclinical chorioamnoitis is one of the most important challenges to choose proper time for termination of pregnancy before established infection with all its health hazards [9].

CRP and TLC are the most widely used marker for early detection of subclinical chorioamnoitis which used with many drawbacks like the presence of undercurrent infection which may change the results of these tests making its interpretation is very difficult [7].

New markers were used, and the most available are N/L ratio and $\mathrm{P} / \mathrm{L}$ ratio, in our study we evaluated both in diagnosis of subclinical chorioamnoitis and comparing them with CRP and TLC.

After statistical analysis of the study it showed that P/L ratio had significant relationship with the presence of subclinical chorioamnoitis with specificity $88.68 \%$ and sensitivity $85.71 \%$, so it could be used for screening for the occurrence of subclinical chorioamnoitis in PROM cases with an accuracy $90 \%$.

$\mathrm{N} / \mathrm{L}$ ratio failed to show any significant relationship with the presence of subclinical chorioamnoitis in studied cases, this is may be attributed to the lower sensitivity for diagnosis of subclinical chorioamnoitis (57.14\%) which means that normal test result couldn't rule out the presence of subclinical chorioamnoitis in PROM cases ,on the other hand it had the higher specificity $92.13 \%$ which means that abnormal test level could diagnose the presence of subclinical chorioamnoitis, so the accuracy of diagnosis reach to be $80 \%$.

CRP and TLC failed to show significant relationship with the presence of subclinical chorioamnoitis and both had lower sensitivity, specificity and accuracy, bit CRP shows significant relationship with the presence of postpartum complication, while the other markers failed to show any significant relationship with it.

The role of $\mathrm{N} / \mathrm{L}$ ratio and $\mathrm{P} / \mathrm{L}$ ration in PROM was discussed in many researches, Ozel A, et al. [10]. Toprak E, et al. [11] and Köseoğlu SB, et al. [12]. All demonstrated the significant of N/L ratio In PROM patients by comparing the level of N/L ratio between PROM ceases and a healthy control case.

Also, the role of $\mathrm{P} / \mathrm{L}$ ratio was discussed in many researches, Toprak E, et al. [11] and Ekin A, et al. [13] demonstrated the significance of P/L ratio in PROM patient by comparing the level of $\mathrm{P} / \mathrm{L}$ ratio between PROM cases and a healthy control case.

In contrary Dundar B, et al. [14] showed that mean Neutrophil count in patients was 8.7 (4.3:26.2) and in control was 7.9 (3:15.4) but with insignificant relationship between both group with p-value 0.12 . Also, they found the platelet count was 217 (136:360) lower in patients group than control 220 (112:490) but insignificant differences between both groups with p-value 0.90 and this may be explained by choosing the patients and taking blood samples immediately after membranes rupturing.

In many aspects we agreed with Liyin $Q$ et al. [15] who investigated the diagnostic value of maternal peripheral blood 
platelet to white blood cell ratio (PLT/WBC) and platelet (PLT) counts for diagnosis of histological chorioamnoitis (HCA) over 400 patients with preterm birth they found that PLT count, PLT/ WBC, and CRP $(\mathrm{P}<0.05)$ were significantly increased in HCA group compared with non HCA group however neutrophil count, WBC count, and procalcitonin show no significant differences, and they Concluded that Platelet count and PLT/WBC is a potential biomarker for detection of HCA.

The only difference is the significance of CRP found in that study which because they did not exclude the cases with clinical chorioamnoitis, and of course all the biomarkers increase in clinical chorioamnoitis.

We also agreed with Toperak E, et al. [11] who investigated the relationship between the platelet-to-lymphocyte ratio (PLR) and N/L ratio with preterm premature membrane rupture on 121 pregnant women with PPROM and 96 age-matched pregnant women with spontaneous preterm labor, they found that The P/L ratio and N/L ratios were both significantly higher in the PPROM group $(p<0.001)$ and also concluded that both markers could be used for the early diagnosis of PPROM, which can help to determine the appropriate waiting time for delivery .

Our study agrees with a large met analysis study by Claudia P, et al. [16], they studied the efficacy of may diagnostic tests for diagnosis of histologic chorioamnoitis in patients at more than 20 weeks of gestation, by analysis of 29 articles and they concluded that CRP and maternal leukocytosis, had a low sensitivity and specificity for diagnosis of subclinical chorioamnoitis.

In conclusion $\mathrm{P} / \mathrm{L}$ ratio and $\mathrm{N} / \mathrm{L}$ ratio are available cheap markers for detection of subclinical chorioamnoitis a, and they show more specificity and accuracy than CRP and TLC in detection of subclinical chorioamnoitis, also P/L ration had higher sensitivity.

\section{Acknowledgement}

None.

\section{Conflict of Interest}

Authors declare no conflict of interest.

\section{References}

1. Deering SH, Patel N, Spong CY, Pezzullo JC, Ghidini A (2007) Fetal growth after preterm premature rupture of membranes: is it related to amniotic fluid volume? Matern Fetal Neonatal Med 20(5): 397-400.

2. Caughey AB , Robinson JN, Norwitz ER (2008) Contemporary diagnosis and management of preterm premature rupture of membranes. Rev
Obstet Gynecol 1(1): 11-22.

3. Medina TM, Hill DA (2006) Preterm premature rupture of membranes : diagnosis and management. Am Fam Physician 73(4): 659-664.

4. Parry S, Strauses JF (1998) Premature rupture of the membranes. N Engl J Med 338(10): 663-670.

5. Seaward PG, Hannah ME, Myhr TL, Farine D, Ohlsson A, et al. (1997) International Multicentre Term Prelabor Rupture of Membranes Study .Evaluation of predictors of clinical chorioamnionitis and post -partum fever in patients with prelabor rupture of membranes at term. Am J Obstet Gynecol 177(5):1024-1029.

6. Goldenberg RL, Hauth JC, Andrews WW (2000) Intrauterine infection and preterm delivery. N Engl J Med 342(20): 1500-1504.

7. Bańkowska EM, Leibschang J, Pawłowska A (2003) Usefulness of determination of granulocyte elastase plasma level ,c-reactive protein and white blood cell count in prediction in intrauterine infection in pregnant women after PROM. Ginekol Pol 74(10):1037-1043.

8. Turkman K, Erdur FM , Ozcicek A, Akbas EM, Ozbicer A, et al. (2013) Platelet to lymphocyte ratio better predicts inflammation than neutrophil -to lymphocyte ratio in end-stage renal disease patient. Hemodial Int 17(3): 391-396.

9. Garite TJ (2001) Management of premature rupture of membranes. Clin Perinatol 28(4): 837-847.

10. Ozel A, Davutoglu EA, Yurtkal A, Madazli R (2020) How do platelet-tolymphocyte ratio and neutrophil-to-lymphocyte ratio change in women with preterm premature rupture of membranes, and threaten preterm labour? J Obstet Gynaecol 40(2): 195-199.

11. Toprak E, Bozkurt M, Dinçgez Çakmak B (2017) Platelet-to-lymphocyte ratio: A new inflammatory marker for the diagnosis of preterm premature rupture of membranes. J Turk Ger Gynecol Assoc 18(3): 122126.

12. Köseoğlu SB, Guzel AI, Deveer R (2014) Maternal serum amyloid A levels in pregnancies complicated with preterm prelabour rupture of membranes. Ginekol Pol 85(7): 516-520.

13. Ekin A, Gezer C, Taner, Ozeren M, Uyar I, et al. (2014) Risk factors and perinatal outcomes associated with latency in preterm premature rupture of membranes between 24 and 34 weeks of gestation. Arch Gynecol Obstet 290(3): 449-455.

14. Dundar B, Cakmak BD, Ozgen G, Tasgoz FN, Guclu T, et al. (2018) Platelet indices in preterm premature rupture of membranes and their relation with adverse neonatal outcomes. J Obstet Gynaecol 44(1): 67-73.

15. Liyin Q,Mian P, Ronglian Zh, Kunhai R (2019) Maternal peripheral blood platelet to white blood cell ratio and platelet count as potential diagnostic markers of histological chorioamnionitis-related spontaneous preterm birth. Journal of Clinical Laboratory Analysis 33(4): 1-6.

16. Claudia PC, Javier F, Herney AG (2018) Validation of diagnostic tests for histologic chorioamnionitis: Systematic review and meta-analysis. Eur J Obstet Gynecol Reprod Biol 228: 13-26. 